\title{
Borderline Ovarian Serous Tumor
}

National Cancer Institute

\section{Source}

National Cancer Institute. Borderline Ovarian Serous Tumor. NCI Thesaurus. Code C5226.

A low grade serous epithelial neoplasm arising from the ovary. It is characterized by an atypical proliferation of serous-type epithelial cells without evidence of stromal invasion. It is often asymptomatic but rarely it may present with abdominal pain or abdominal enlargement due to rupture or torsion. 